\title{
Minimizing Communication Cost in Fine-Grain Partitioning of Sparse Matrices ${ }^{\star}$
}

\author{
Bora Uçar and Cevdet Aykanat \\ Department of Computer Engineering, Bilkent University, 06800, Ankara, Turkey \\ \{ubora, aykanat\}@cs. bilkent.edu.tr
}

\begin{abstract}
We show a two-phase approach for minimizing various communication-cost metrics in fine-grain partitioning of sparse matrices for parallel processing. In the first phase, we obtain a partitioning with the existing tools on the matrix to determine computational loads of the processor. In the second phase, we try to minimize the communicationcost metrics. For this purpose, we develop communication-hypergraph and partitioning models. We experimentally evaluate the contributions on a PC cluster.
\end{abstract}

\section{Introduction}

Repeated matrix-vector multiplications $(\mathrm{SpMxV}) y=A x$ that involve the same large, sparse, structurally symmetric or nonsymmetric square or rectangular matrix are kernel operations in various iterative solvers. Efficient parallelization of these solvers requires matrix $A$ to be partitioned among the processors in such a way that communication overhead is kept low while maintaining computational load balance. Because of possible dense vector operations, some of these methods require symmetric partitioning on the input and output vectors, i.e, conformal partitioning on $x$ and $y$. However, quite a few of these methods allow unsymmetric partitionings, i.e., $x$ and $y$ may have different partitionings. The standard graph partitioning model has been widely used for one-dimensional (1D) partitioning of sparse matrices. Recently, Çatalyürek and Aykanat [34] and others [9:10 11] demonstrated some flaws and limitations of this model and developed alternatives. As noted in [10], most of the existing models consider minimizing the total communication volume. Depending on the machine architecture and the problem characteristics, communication overhead due to message latency may be a bottleneck as well [8]. Furthermore, maximum communication volume and latency handled by a single processor may also have crucial impacts on the parallel performance. In our previous work [17], we addressed these four communication-cost metrics in 1D partitioning of sparse matrices.

The literature on $2 \mathrm{D}$ matrix partitioning is rare. The $2 \mathrm{D}$ checkerboard partitioning approaches proposed in [12,15,16] are suitable for dense matrices or sparse matrices with structured nonzero patterns that are difficult to exploit. In

* This work was partially supported by The Scientific and Technical Research Council of Turkey under project EEEAG-199E013 
particular, these approaches do not exploit sparsity to reduce the communication volume. Çatalyürek and Aykanat 367. proposed hypergraph models for $2 \mathrm{D}$ coarse-grain and fine-grain sparse matrix partitionings. In the coarse-grain model, a matrix is partitioned in a checkerboard-like style. In the fine-grain model, a matrix is partitioned on nonzero basis. The fine-grain model is reported to achieve better partitionings than the other models in terms of the total communication volume metric [6]. However, it also generates worse partitionings than the other models in terms of the total number of messages metric [6]. In this work, we adopt our two phase approach [17] to minimize the four communication-cost metrics in the fine-grain partitioning of sparse matrices. We show how to apply our communication hypergraph model to obtain unsymmetric partitioning and develop novel models to obtain symmetric partitioning.

\section{Preliminaries}

A hypergraph $\mathcal{H}=(\mathcal{V}, \mathcal{N})$ is defined as a set of vertices $\mathcal{V}$ and a set of nets. Every net $n_{i}$ is a subset of vertices. Let $d_{j}$ denote the number of nets containing a vertex $v_{j}$. Weights can be associated with vertices. $\Pi=\left\{\mathcal{V}_{1}, \cdots, \mathcal{V}_{K}\right\}$ is a $K$-way vertex partition of $\mathcal{H}=(\mathcal{V}, \mathcal{N})$ if each part $\mathcal{V}_{k}$ is non-empty, parts are pairwise disjoint, and the union of parts gives $\mathcal{V}$. In $\Pi$, a net is said to connect a part if it has at least one vertex in that part. Connectivity $\lambda_{i}$ of a net $n_{i}$ denotes the number of parts connected by $n_{i}$. A net $n_{j}$ is said to be cut if $\lambda_{j}>1$ and uncut otherwise. The set of cut and uncut nets are called external and internal nets, respectively. In $\Pi$, the weight of a part is the sum of the weights of the vertices in that part. In the hypergraph partitioning problem, the objective is to minimize the cutsize:

$$
\text { cutsize }(\Pi)=\sum_{n_{i} \in \mathcal{N}}\left(\lambda_{i}-1\right) \text {. }
$$

This objective is referred to as the connectivity-1 cutsize metric [14. The partitioning constraint is to satisfy a balancing constraint on the part weights, i.e.,

$$
W_{\max } \leq(1+\epsilon) W_{\text {avg }}
$$

where $W_{\max }$ is the weight of the part with the maximum weight and $W_{\text {avg }}$ is the average part weight, and $\epsilon$ is an imbalance ratio. This problem is NP-hard [14].

A recent variant of the above problem is called multi-constraint hypergraph partitioning 3,7,13. In this problem, a vertex has a vector of weights. The partitioning objective is the same as above, however, the partitioning constraint is to satisfy a set of balancing constraints, one for each type of the weights.

In the fine-grain hypergraph model of Çatalyürek and Aykanat [6], an $M \times N$ matrix $A$ with $Z$ nonzeros is represented as a hypergraph $\mathcal{H}=(\mathcal{V}, \mathcal{N})$ with $|\mathcal{V}|=Z$ vertices and $|\mathcal{N}|=M+N$ nets for $2 \mathrm{D}$ partitioning. There exists one vertex $v_{i j}$ for each nonzero $a_{i j}$. There exists one net $m_{i}$ for each row $i$ and one net $n_{j}$ for each column $j$. Each row-net $m_{i}$ and column-net $n_{j}$ contain all vertices $v_{i *}$ and $v_{* j}$, respectively. Each vertex $v_{i j}$ corresponds to scalar multiplication $a_{i j} x_{j}$. Hence, the computational weight associated with a vertex is 1 . Each row-net $m_{i}$ represents the dependency of $y_{i}$ on the scalar multiplications with $a_{i *}$ 's. Each 
column-net $n_{j}$ represents the dependency of scalar multiplications with $a_{* j}$ 's on $x_{j}$. With this model, the problem of $2 \mathrm{D}$ partitioning a matrix among $K$ processors reduces to the $K$-way hypergraph partitioning problem. In this model, minimizing the cutsize while maintaining the balance on the part weights corresponds to minimizing the total communication volume and maintaining the balance on the computational loads of the processors. An external column-net represents the communication volume requirement on a $x$-vector entry. This communication occurs in expand phase, just before the scalar multiplications. An external row-net represents the communication volume requirement on a $y$-vector entry. This communication occurs in fold phase, just after the scalar multiplications. Çatalyürek and Aykanat [6] assign the responsibility of expanding $x_{i}$ and folding $y_{i}$ to the processor that holds $a_{i i}$ to obtain symmetric partitioning. Note that for the unsymmetric partitioning case, one can assign $x_{i}$ to any processor holding a nonzero in column $i$ without any additional communication-volume overhead. A similar opportunity exists for $y_{i}$. In the symmetric partitioning case, however, $x_{i}$ and $y_{i}$ may be assigned to a processor holding nonzeros both in the row and column $i$. In this work, we try to exploit the freedom in assigning vector elements to address the four communication-cost metrics.

A $10 \times 10$ matrix with 37 nonzeros and its 4-way fine-grain partitioning is given in Fig. 1(a). In the figure, the partitioning is given by the processor numbers for each nonzero. The computational load balance is achieved by assigning $9,10,9$, and 9 nonzeros to processors in order.

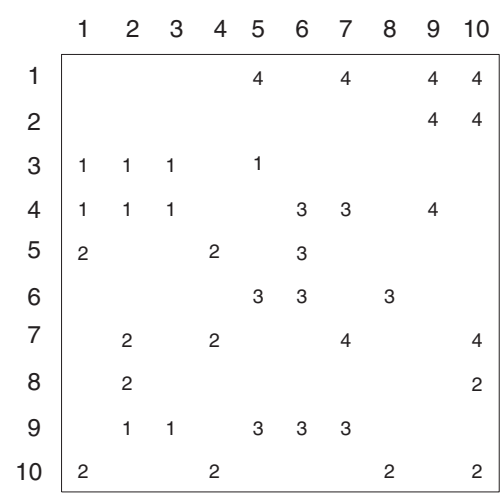

(a)

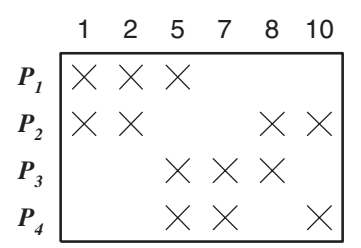

(b)

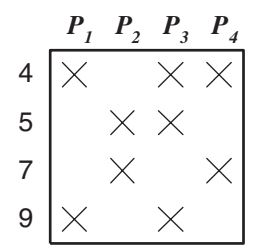

(c)

Fig. 1. (a) A $10 \times 10$ matrix and a 4-way partitioning, (b) communication matrix $C_{x}$, and (c) communication matrix $C_{y}$ 


\section{Minimizing the Communication Cost}

Given a $K$-way fine-grain partitioning of a matrix, we identify two sets of rows and columns; internal and coupling. The internal rows or columns have nonzeros only in one part. The coupling rows or columns have nonzeros in more than one part. The set of $x$-vector entries that are associated with the coupling columns, referred to here as $x_{C}$, necessitate communication. Similarly, the set of $y$-vector entries that are associated with the coupling rows, referred to here as $y_{C}$, necessitate communication. Note that when symmetric partitioning requirement arises we add to $x_{C}$ those $x$-vector entries whose corresponding entries are in $y_{C}$ and vice versa. The proposed approach considers partitioning of these $x_{C}$ and $y_{C}$ vector entries to reduce the total message count and the maximum communication volume per processor. The other vector entries are needed by only one processor and should be assigned to the respective processors to avoid redundant communication. The approach may increase the total volume of communication of the given partitioning by at most $\max \left\{\left|x_{C}\right|,\left|y_{C}\right|\right\}$. We propose constructing two matrices $C_{x}$ and $C_{y}$, referred to here as communication matrices, that summarize the communication on $x$ - and $y$-vector entries, respectively. Matrix $C_{x}$ has $K$ rows and $\left|x_{C}\right|$ columns. For each row $k$ we insert a nonzero in column $j$ if processor $P_{k}$ has nonzeros in column corresponding to $x_{C}[j]$ in the fine-grain partitioning. Hence, the rows of $C_{x}$ correspond to processors in such a way that the nonzeros in the row $k$ identify the subset of $x_{C}$-vector entries that are needed by processor $P_{k}$. Matrix $C_{y}$ is constructed similarly. This time we put processors in columns and $y_{C}$ entries in rows. Figure 1(b) and (c) show communication matrices $C_{x}$ and $C_{y}$ for the sample matrix given in (a).

\subsection{Unsymmetric Partitioning Model}

We use row-net and column-net hypergraph models for representing $C_{x}$ and $C_{y}$, respectively. In the row-net hypergraph model, matrix $C_{x}$ is represented as hypergraph $\mathcal{H}_{x}$ for columnwise partitioning. The vertex and net sets of $\mathcal{H}_{x}$ correspond to the columns and rows of matrix $C_{x}$, respectively. There exist one vertex $v_{j}$ and one net $n_{i}$ for each column $j$ and row $i$, respectively. Net $n_{i}$ contains the vertices corresponding to the columns which have a nonzero in row $i$. That is, $v_{j} \in n_{i}$ if $C_{x}[i, j] \neq 0$. In the column-net hypergraph model $\mathcal{H}_{y}$ of $C_{y}$, the vertex and net sets correspond to the rows and columns of the matrix $C_{y}$, respectively, with similar construction. Figure 2(a) and (b) show communication hypergraphs $\mathcal{H}_{x}$ and $\mathcal{H}_{y}$.

A $K$-way partition on the vertices of $\mathcal{H}_{x}$ induces a processor assignment for the expand operations. Similarly, a $K$-way partition on the vertices of $\mathcal{H}_{y}$ induces a processor assignment for the fold operations. In unsymmetric partitioning case, these two assignment can be found independently. In [17] we showed how to obtain such independent partitionings in order to minimize the four communication-cost metrics. The results of that work are immediately applicable to this case. 


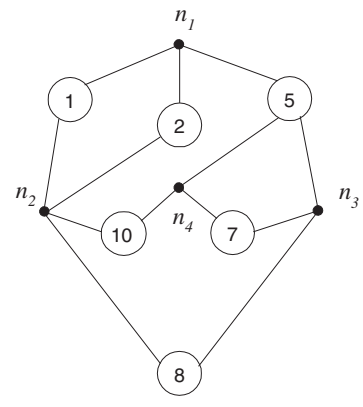

(a)

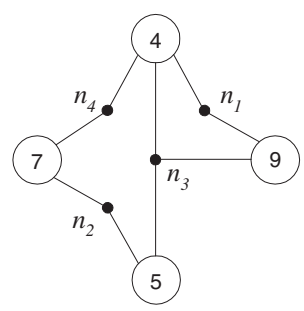

(b)

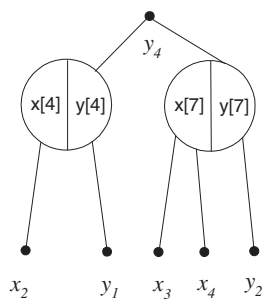

(c)

Fig. 2. Communication hypergraphs: (a) $\mathcal{H}_{x}$, (b) $\mathcal{H}_{y}$, and (c) a portion of $\mathcal{H}$

\subsection{Symmetric Partitioning Model}

When we require symmetric partitioning on vectors $x$ and $y$, the partitionings on $\mathcal{H}_{x}$ and $\mathcal{H}_{y}$ can not be obtained independently. Therefore, we combine hypergraphs $\mathcal{H}_{x}$ and $\mathcal{H}_{y}$ into a single hypergraph $\mathcal{H}$ as follows. For each part $P_{k}$, we create two nets $x_{k}$ and $y_{k}$. For each $x_{C}[i]$ and $y_{C}[i]$ pair, we create a single vertex $v_{i}$. For each net $x_{k}$, we insert $v_{i}$ into its vertex list if processor $P_{k}$ needs $x_{C}[i]$. For each $y_{k}$, we insert $v_{j}$ into its vertex list if processor $P_{k}$ contributes to $y_{C}[j]$. We show vertices $v_{4}$ and $v_{7}$ of $\mathcal{H}$ in Fig. 2 (c). Since communication occurs in two distinct phases, vertices have two weights associated with them. The first weight of a vertex $v_{i}$ is the communication volume requirement incurred by $x_{C}[i]$; hence we associate weight $d_{i}-1$ with the vertex $v_{i}$. The second weight of a vertex $v_{i}$ is the communication volume requirement incurred by $y_{C}[i]$; as in [17] we associate a unit weight of 1 with each $v_{i}$.

In a $K$-way partition of $\mathcal{H}$, an $x_{k}$-type net connecting $\lambda_{x k}$ parts necessitates $\lambda_{x k}-1$ messages to be sent to processor $P_{k}$ during the expand phase. The sum of these values thus represents the total number of messages sent during the expand phase. Similarly, a $y_{k}$-type net connecting $\lambda_{y k}$ parts necessitates $\lambda_{y k}-1$ messages to be sent by $P_{k}$ during the fold phase. The sum of these values represents the total number of messages sent during the fold phase. The sum of the connectivity -1 values for all nets thus represents the total number of messages. Therefore, by minimizing the objective function in Eq. 1, partitioning $\mathcal{H}$ minimizes the total number of messages. The vertices in part $\mathcal{V}_{k}$ represent the $x$-vector entries to be expanded and the respective $y$-vector entries to be folded by processor $P_{k}$. The load of the expand operations are exactly represented by the first components of the vertex weights if for each $v_{i} \in \mathcal{V}_{k}$ we have $v_{i} \in$ $x_{k}$. If, however, $v_{i} \notin x_{k}$, the weight of a vertex for the expand phase will be one less than the required. We hope these shortages to occur, in some extent, for every processor to cancel the diverse effects on the communication-volume load balance. The weighting scheme for the fold operations is adopted with the rationale that every $y_{C}[i]$ assigned to a processor $P_{k}$ will relieve $P_{k}$ from sending 
a unit-volume message. If the net sizes are close to each other then this scheme will prove to be a reasonable one. As a result, balancing part sizes for the two set of weights, e.g., satisfying Eq. 2, will relate to balancing the communicationvolume loads of processors in the expand and the fold phases, separately. For the minimization of the maximum number of messages per processor metric we do not spend explicit effort. We merely rely on its loose correlation with the total number of messages metric.

In the above discussion, each net is associated with a certain part and hence a processor. This association is not defined in the standard hypergraph partitioning problem. We can enforce this association by adding $K$ special vertices, one for each processor $P_{k}$, and inserting those vertices to the nets $x_{k}$ and $y_{k}$. Fixing those special vertices to the respective parts and using partitioning with fixed vertices feature of hypergraph partitioning tools [1,5] we can obtain the specified partitioning on $\mathcal{H}$. However, existing tools do not handle fixed vertices within multi-constraint framework. Therefore, instead of obtaining balance on the communication-volume loads of processors in the expand and the fold phases separately, we add up the weights of vertices and try to obtain balance on aggregate communication-volume loads of processors.

\section{Experiments}

We have conducted experiments on the matrices given in Table 1 . In the table, $N$ and $N N Z$ show, respectively, the dimension of the matrix and the number of nonzeros. The Srl. Time column lists the timings for serial SpMxV operations in milliseconds. We used PaToH [5] library to obtain 24-way fine-grain partitionings on the test matrices. In all partitioning instances, the computational-load imbalance were below 7 percent. Part. Mthd give the partitioning method applied: PTH refers to the fine-grain partitioning of Çatalyürek and Aykanat [6], CHy refers to partitioning the communication hypergraphs with fixed vertices and aggregate vertex weights. For these two methods, we give timings under column Part.Time, in seconds. For each partitioning method, we dissect the communication requirements into the expand and fold phases. For each phase, we give the total communication volume, the maximum communication volume handled by a single processor, the total number of messages, and the maximum number of messages per processor under columns $\mathrm{tV}, \mathrm{xV}$, tM, and $\mathrm{xM}$, respectively. In order to see whether the improvements achieved by method CHy in the given performance metrics hold in practice, we also give timings, the best among 20 runs, for parallel SpMxV operations, in milliseconds, under column Prll. Time. All timings are obtained on machines equipped with $400 \mathrm{MHz}$ Intel Pentium II processor and 64 MB RAM running Linux kernel 2.4.14 and Debian GNU/Linux 3.0 distribution. The parallel $\mathrm{SpMxV}$ routines are implemented using LAM/MPI 6.5.6 2]. To compare our method against PTH, we opted for obtaining symmetric partitioning. For each matrix, we run PTH 20 times starting from different random seeds and selected the partition which gives the minimum in total communication volume metric. Then, we constructed the communication hypergraph with respect to PTH's best partitioning and run CHy 20 times, again starting from 
different random seeds, and selected the partition which gives the minimum in total number of messages metric. Timings for these partitioning methods are for a single run. In all cases, running CHy adds at most half of the time required by PTH to the framework of fine-grain partitioning.

In all of the partitioning instances, CHy reduces the total number of messages to somewhere between 0.47 (fom12) and 0.74 (CO9) of PTH. In all partitioning instances, CHy increases the total communication volume to somewhere between 1.32 (creb) and 1.86 ( $p d s 20$ ) of PTH. This is expected, because a vertex $v_{i}$ may be assigned to a part $\mathcal{V}_{k}$ while $P_{k}$ does not need any of $x_{C}[i]$ or $y_{C}[i]$. However, reductions in parallel running times are seen for all matrices except lpl1. The highest speed-up achieved by PTH and CHy is 5.96 and 6.38, respectively, on fxm3.

Table 1. Communication patterns and running times for 24-way parallel SpMxV

\begin{tabular}{|c|c|c|c|c|c|c|c|c|c|c|}
\hline \multirow[t]{2}{*}{ Matrix } & Size & \multicolumn{2}{|c|}{ Part. } & \multicolumn{2}{|c|}{ Expand Phase } & \multicolumn{3}{|c|}{ Fold Phase } & \multirow{2}{*}{$\begin{array}{c}\text { Prll. } \\
\text { Time }\end{array}$} & \multirow{2}{*}{$\begin{array}{l}\text { Srl. } \\
\text { Time }\end{array}$} \\
\hline & $N$ & & Time & tV & $\begin{array}{lll}x V & t M & x M\end{array}$ & tV & $\mathrm{xV}$ & tM $\quad \mathrm{xM}$ & & \\
\hline \multirow[t]{2}{*}{$\mathrm{CO} 9$} & 0789 & PTH & 11.43 & 2477 & 2429021 & 4889 & 473 & 35822 & 4.55 & $19-1$ \\
\hline & & Iy & & & & 7367 & 714 & 59 & 05 & \\
\hline \multirow[t]{2}{*}{ reb } & 06 & PTH & 26.71 & 9344 & 5049023 & 12660 & 871 & 50423 & .64 & \\
\hline & & CHy & 2.51 & 47 & 3 & 57 & 1068 & 20 & 92 & \\
\hline \multirow[t]{2}{*}{$\mathrm{x} 3 \mathrm{~s} 1$} & 857 & PTH & 88 & 64 & 6023 & 34 & 1762 & 35620 & 39 & \\
\hline & & $\mathrm{CHy}$ & 88 & 7 & 23 & 47 & 52 & 10 & 91 & \\
\hline \multirow[t]{2}{*}{$\mathrm{om} 12$} & $=2$ & $\Gamma \mathrm{H}$ & 77 & 09 & 55922823 & 8 & 11 & 22813 & $0:$ & \\
\hline & & CHy & 76 & 13 & $983 \quad 96$ & 2 & 1541 & 1198 & 03 & \\
\hline \multirow[t]{2}{*}{$\mathrm{sm} 3$} & 526 & PTH & 37.73 & 3 & 27921 & 2662 & 282 & 23617 & $39:$ & \\
\hline & & CHy & & 3299 & 2151 & 4027 & 456 & - & .97 & \\
\hline \multirow[t]{2}{*}{ lpl1 } & 217 & PTH & 27.04 & 7646 & 1062 & 13752 & 961 & 25317 & 5.73 & 29 \\
\hline & & CHy & & & 8921 & 20 & 1 & 5612 & .83 & \\
\hline \multirow[t]{2}{*}{$\bmod 2$} & 910 & PTH & 32.83 & 15 & 23 & 9421 & 35 & 22 & .92 & 30.8 \\
\hline & & CHy & & & 56 & 2 & 17 & 19817 & .92 & \\
\hline \multirow[t]{2}{*}{ ds 20} & 96 & PTH & 18.65 & 5373 & 5572 & 13548 & 956 & 31719 & 5.23 & 7 \\
\hline & & CHy & & & 941 & & & 113 & 95 & \\
\hline \multirow[t]{2}{*}{ pltex } & 26894269736 & PT & 14.29 & & 17216716 & 85 & 508 & 27320 & 4.27 & 14. \\
\hline & & CHy & & & $\begin{array}{lll}311 & 89 & 16\end{array}$ & 828 & 782 & 13910 & 63 & \\
\hline \multirow[t]{2}{*}{ orld } & 4506582064 & PTH & 30.84 & 4934 & 79430023 & 9710 & 1295 & 31623 & 7.35 & 2 \\
\hline & & CHy & 2.50 & 10679 & 656181 & 485 & & 20518 & 6.05 & \\
\hline
\end{tabular}

\section{Conclusion and Future Work}

We showed a two-phase approach that encapsulates various communication-cost metrics in 2D partitioning of sparse matrices. We developed models to obtain symmetric and unsymmetric partitioning on input and output vectors. We tested performance of the proposed models on practical implementations. In this work, a sophisticated hypergraph partitioning tool that can handle fixed vertices in the context of multi-constraint partitioning was needed. Since the existing tools do not handle this type of partitioning, we are considering to develop such a method. 


\section{References}

1. Charles J. Alpert, Andrew E. Caldwell, Andrew B. Kahng, and Igor L. Markov. Hypergraph partitioning with fixed vertices. IEEE Transactions on ComputerAided Design, 19(2):267-272, 2000.

2. Greg Burns, Raja Daoud, and James Vaigl. LAM: an open cluster environment for MPI. In John W. Ross, editor, Proceedings of Supercomputing Symposium '94, pages 379-386. University of Toronto, 1994.

3. Ü. V. Çatalyürek. Hypergraph Models for Sparse Matrix Partitioning and Reordering. PhD thesis, Bilkent Univ., Computer Eng. and Information Sci., Nov 1999.

4. Ü. V. Çatalyürek and C. Aykanat. Hypergraph-partitioning based decomposition for parallel sparse-matrix vector multiplication. IEEE Transactions on Parallel and Distributed Systems, 10(7):673-693, 1999.

5. Ü. V. Çatalyürek and C. Aykanat. PaToH: A multilevel hypergraph partitioning tool, ver. 3.0. Tech. Rep. BU-CE-9915, Computer Eng. Dept., Bilkent Univ., 1999.

6. Ü. V. Çatalyürek and Cevdet Aykanat. A fine-grain hypergraph model for $2 \mathrm{~d}$ decomposition of sparse matrices. In Proceedings of International Parallel and Distributed Processing Symposium (IPDPS), 8th International Workshop on Solving Irregularly structured Problems in Parallel (Irregular 2001), April 2001.

7. Ü. V. Çatalyürek and Cevdet Aykanat. A hypergraph-partitioning approach for coarse-grain decomposition. In Proceedings of Scientific Computing 2001 (SC2001), pages 10-16, Denver, Colorado, November 2001.

8. J. J. Dongarra and T. H. Dunigan. Message-passing performance of various computers. Concurrency-Practice and Experience, 9(10):915-926, 1997.

9. B. Hendrickson. Graph partitioning and parallel solvers: has the emperor no clothes? Lecture Notes in Computer Science, 1457:218-225, 1998.

10. B. Hendrickson and T. G. Kolda. Graph partitioning models for parallel computing. Parallel Computing, 26:1519-1534, 2000.

11. B. Hendrickson and T. G. Kolda. Partitioning rectangular and structurally unsymmetric sparse matrices for parallel processing. SIAM Journal on Scientific Computing, 21(6):2048-2072, 2000.

12. B. Hendrickson, R. Leland, and S. Plimpton. An efficient parallel algorithm for matrix-vector multiplication. Int. J. High Speed Comput., 7(1):73-88, 1995.

13. G. Karypis and V. Kumar. Multilevel algorithms for multi-constraint hypergraph partitioning. Tech. Rep. 99-034, University of Minnesota, Dept. Computer Science/Army HPC Research Center, Minneapolis, MN 55455, November 1998.

14. T. Lengauer. Combinatorial Algorithms for Integrated Circuit Layout. WileyTeubner, Chichester, U.K., 1990.

15. J. G. Lewis, D. G. Payne, and R. A. van de Geijn. Matrix-vector multiplication and conjugate gradient algorithms on distributed memory computers. In Proceedings of the Scalable High Performance Computing Conference, 1994.

16. A. T. Ogielski and W. Aiello. Sparse matrix computations on parallel processor arrays. SIAM Journal on Numerical Analysis, 1993.

17. Bora Uçar and Cevdet Aykanat. Encapsulating multiple communication-cost metrics in partitioning sparse rectangular matrices for parallel matrix-vector multiplies. Siam J. Sci. Comput., Submitted to, 2002. 